\title{
Suffering
}

\author{
Bert Gordijn ${ }^{1} \cdot$ Henk ten Have ${ }^{2}$
}

Published online: 25 July 2020

(c) Springer Nature B.V. 2020

Suffering - in its various forms - has always been a fundamental concern in philosophy, theology and literature. More recently, it has become the subject matter of highly contextualized analyses in medical end-of-life decision making. The first three papers in the current issue contribute to this strand of research. More specifically, they focus on the following three questions: (1) What is suffering? (2) What should medicine do about suffering? (3) What is the role of suffering when justifying medical decisions at the end of life?

\section{What is suffering?}

Svenaeus (2020) develops a broad phenomenological account of suffering in terms of embodied moods and shared being-in-the-world. It transcends the bodily realm, as does its counterpart, human flourishing. Thus, suffering can only properly and comprehensively be understood when taking into account life plans, narratives as well as core values. Accordingly, it can materialize in quite different forms: bodily agonies, a loss of engagement in the world together with others, or the inability to realize core life values.

Streeck (2020) accepts the way suffering has been conceptualised in palliative care from its early days, i.e. as a complex phenomenon involving bodily, psychological, social and spiritual or existential dimensions.

Ahlzen (2020) regards authenticity as essential for a better understanding of suffering. An authentic will is expressed in behaviour and decisions rooted in convictions and values that a person on reflection identifies as their own. Unfortunately, assessing authenticity is notoriously problematic. Also, the relation between authenticity and suffering is complicated. If we lose our authenticity, i.e. if we cannot connect to our deeply held convictions, access our core values and

\footnotetext{
Bert Gordijn

bert.gordijn@dcu.ie

Dublin City University, Dublin, Ireland

2 Duquesne University, Pittsburgh, USA
}

realise our true purpose, it seems difficult to fathom how life can still be worth living. Nevertheless, sometimes severely demented patients who have clearly lost their authenticity still seem to be able to have good lives. So inauthenticity does not automatically lead to a complete loss of quality of life.

\section{What should medicine do about suffering?}

Svenaeus (2020) holds that doctors should attempt to understand the suffering of their patients not only as caused by some bodily dysfunction but as a subjective experience. The latter can only be accomplished through empathy, narrative and dialogue. This is especially important in end-of-life care. Though palliative care has achieved improved treatment of suffering narrowly understood, as caused by all kinds of bodily dysfunctions, medicine as a whole has not made great strides in addressing suffering properly understood, i.e. from a broad phenomenologically informed perspective.

Streeck (2020) maintains that the strong focus on prevention and relief of suffering within palliative care might have unintended but seriously negative consequences. In particular, she sketches two different pathways in which this might play out. First, if attempts at thwarting or alleviating suffering happen to be unsuccessful, there are only two options left: terminal sedation or just continuing the attempts already started. Terminal sedation renders patients in an unconscious state, it triggers fierce discussions about the precise demarcation with euthanasia, and its increased practise might lead to a greater acceptance of physician assisted suicide and euthanasia, as these are also motivated by the elimination of suffering. Continuing attempts at mitigating suffering that have thus far been unsuccessful, on the other hand, might prove to be futile. In addition, it could put mounting pressure on patients to match the caregivers' expectations, thus increasing the suffering. Second, if patients-for whatever reasons, e.g. not wanting to be sedated or wishing to make practical arrangements for imminent death-wish to tolerate a certain kind of suffering instead of having it treated, 
continued efforts to suppress this suffering might in truth counteract patients' aims and desires. After all, patients being in a vulnerable position might feel pressure to accept the palliative care treatment offered, which might thus actually lower their quality of life as perceived from the patients' point of view.

Ahlzen (2020) endorses the common-sense view that medicine should attempt to alleviate suffering. In addition, he acknowledges that medicine in general and palliative care in particular have gained progress in tackling suffering. Yet, all suffering at the end of life has obviously not been eradicated.

\section{What is the role of suffering when justifying medical decisions at the end of life?}

Svenaeus (2020) points out that suffering has been advanced as a justification for physician assisted suicide and euthanasia. Thoughtfully, Svenaeus himself seems to be openunder certain circumstances - to assisted suicide as a measure to avoid extreme suffering. However, he also argues that institutionalisation or legalisation of physician assisted suicide might boost an approach to human flourishing and suffering dominated by considerations of utility and productivity, which might negatively impact the sense-making resources of dying persons.

Streeck (2020) sees a similarity between palliative care proponents and advocates of assisted dying in that they are both very much focused on eliminating suffering. As a result, she imagines that a strong and exclusive anti-suffering focus within palliative care might even play into the hands of the assisted dying camp; it could lead to an even stronger taboo on suffering, thus strengthening the main argument of the champions of assisted dying, i.e. to offer an immediate and effective remedy against unbearable or intolerable suffering.

Ahlzen (2020) also highlights the increased acceptance of physician assisted suicide and euthanasia in Western Europe and North America, which has been reflected in legislative changes in several countries. He acknowledges that suffering and autonomous patient requests have played a pivotal role in the usual defence of the practice of assisted dying. In addition, he advances authenticity as an essential further element in any such justificatory framework. Because of the complicated nature of the concept of authenticity, however, he does not reach any definite conclusions on the precise character of its justificatory role.

\section{Ambivalences}

The three papers above call attention to the complicated nature of suffering in medicine and end-of-life decision making. More specifically, they call attention to three ambivalences. First, they highlight the difference between a scientific third-person point of view and a phenomenological first-person approach to suffering. These two ways of understanding each seem to be based on their own distinctive modus operandi. Traditionally, medical education has very much focused on enabling doctors to engage with suffering in the scientific manner and has neglected training to use the phenomenological method. Consequently, it is not easy to figure out how to combine and balance these approaches in patient care.

Second, though common-sense moral intuitions suggest that suffering is intrinsically bad, an exclusive focus on its eradication is fraught with problems. While bad in itself, suffering might still have significant instrumental value for patients in certain situations. In addition, suffering and its positive counterparts, i.e. wellbeing, happiness and flourishing, are not the only important values at play in medicine. Besides, autonomy, authenticity, justice and solidarity should also enter into the equation. Medicine should not become too hedonistic in its dealings with suffering.

Third, the justificatory role of authenticity in assisted dying is inconclusive. On the one hand, if a patient request for assisted dying turns out to be inauthentic, this weakens any attempt at justifying a medically assisted death. Understood as an essential element of suffering on the other hand, inauthenticity might strengthen such a justification.

\section{References}

Ahlzen, R. 2020. Suffering, authenticity, and physician assisted suicide. Medicine, Health Care and Philosophy.. https://doi.org/10.1007/ s11019-019-09929-z.

Streeck, N. 2020. Death without distress? The taboo of suffering in palliative care. Medicine, Health Care and Philosophy.. https:// doi.org/10.1007/s11019-019-09921-7.

Svenaeus, F. 2020. To die well: the phenomenology of suffering and end of life ethics. Medicine, Health Care and Philosophy.. https ://doi.org/10.1007/s11019-019-09914-6.

Publisher's Note Springer Nature remains neutral with regard to jurisdictional claims in published maps and institutional affiliations. 